The development of a novel supplemental coding system for investigating the practice elements of attachment-based interventions

Ahmed Riaz Mohamed ${ }^{1,2,4}$, Paula Sterkenburg ${ }^{1,3}$, Esmé van Rensburg $^{2} \&$ Carlo Schuengel $^{1}$

${ }^{1}$ Department of Clinical Child and Family Studies and Amsterdam Public Health Research Institute, Vrije Universiteit, Amsterdam, Netherlands, ${ }^{2}$ Department of Psychology, NorthWest University, Potchefstroom, South Africa, ${ }^{3}$ Department of Psychotherapy, Bartiméus, Doorn, Netherlands, ${ }^{4}$ Department of Psychology, University of Pretoria, Pretoria, South Africa

Author Note:

Corresponding author: Ahmed Riaz Mohamed, Department of Psychology, University of Pretoria, Private Bag X20, Hatfield, 0028, Pretoria, South Africa. Email: 


\begin{abstract}
While research generally demonstrates the efficacy of attachment-based interventions for supporting families and children, less is known about the constituent elements of these interventions and their effects. Existing tools for extracting information about these elements from psychosocial intervention studies do not differentiate between techniques used in attachment-based interventions. Therefore, a novel extension of the PracticeWise coding system was developed for attachment-based interventions. This paper describes the development of this new extension. A systematic literature search of randomized controlled trials of attachment-based interventions (1998-2018) identified 66 publications. This led to $\mathrm{N}$ $=49$ unique attachment-based interventions. Study authors were requested to share the intervention manuals for these trials. Publications or, if available, manuals were initially coded using the standard PracticeWise coding system. Techniques falling under the general definition of a practice element, but not captured by the standard system, formed the basis for a novel set of subcategories. To identify and develop definitions for this novel subset of practice elements, the publications and manuals were carefully examined and initial codes were developed inductively for each intervention, and then discussed to reach consensus. These codes were then applied across all interventions. Through an iterative process, 16 unique practice elements were identified and defined, and clustered into five thematic groupings: Modelling; Parent-Child Interaction (Behavior-focused); Mental Representations (Representation-focused); Education \& Guidance; Other. As an illustration, the Attachment and Biobehavioral Catch-up intervention is described using the novel supplemental practice elements coding system.
\end{abstract}

Keywords: Practice elements; supplemental coding system for attachment-based interventions; distillation and matching model; common elements 


\section{The development of a novel supplemental coding system for investigating the practice elements of attachment-based interventions}

Attachment theory (Ainsworth et al., 1978; Bowlby, 1969/1997) is an influential framework for conceptualizing those aspects of caregiver-child relationships that are associated with social competence as well as emotional and behavioral difficulties across the developmental spectrum (Groh et al., 2017). Insights gleaned from attachment research have given rise to the development of numerous interventions to improve the quality of caregiverchild relationships. A number of these manualized attachment-based intervention programs have been subjected to multiple randomized controlled trials-such as Attachment and Biobehavioral Catch-up (Dozier et al., 2009), Video-Feedback Intervention to Promote Positive Parenting (Juffer et al., 2017), Promoting First Relationships (Oxford et al., 2016) and the Circle of Security (Cassidy et al., 2017), among others. Meta-analyses interrogating the effectiveness of attachment-based interventions conducted over the past two decades confirm a general pattern of effectiveness of such interventions in improving parental sensitivity and quality of parent-child attachment relationships (Bakermans-Kranenburg et al., 2003) as well as attachment disorganization (Bakermans-Kranenburg et al., 2005;

Facompré et al., 2018). Interventions, however, have often been investigated in their entirety (absolute efficacy) or by comparing broad classes of intervention (relative efficacy). Such research, however, does not provide insight into how these interventions work and whether interventions may be optimized for specific populations and contexts (Lemmens et al., 2016). With an increasing number of available attachment-based interventions comes the potential complexity for practitioners to identify the appropriate evidence-based interventions to employ especially when many have demonstrated their efficacy. Manualized protocols that have an evidence-base often have overlapping clinical strategies, which may make it more 
challenging for practitioners who wish to use them to determine the relative appropriateness of any given manual over another for specific clients or contexts (Chorpita et al., 2007). Knowing what specific clinical strategies (practice elements) are common across the body of successful attachment-based interventions - a common elements approach - may enhance the understanding of intervention effectiveness and guide further development and implementation.

Chorpita et al. (2005) propose a Distillation and Matching Model in order to operationalize such a common elements approach to evidence-based practice. According to this approach, clinical work, instead of being viewed as a collection of individually effective manualized interventions, is conceptualized in terms of the component parts that are evident across the corpus of such interventions (Barth et al., 2014), which allows for the formation of sample aggregate profiles of these procedures. According to Chorpita and colleagues (Chorpita et al., 2007), evidence-based treatments share a significant proportion of their clinical techniques with each other. This allows for the contents of intervention manuals to be 'distilled', or separated out into distinct techniques, making it possible to adopt a modular approach to delivering clinical services which allows clinicians to tailor the content of their interventions to match the specific needs of a client or context (Lee et al., 2014). Furthermore, Chorpita and Daleiden (2009) argue that this approach defines a level of analysis (i.e. practice elements) that can be aggregated across intervention manuals and studies thereof. This makes it possible to evaluate, empirically, the inferences made about specific intervention content. The model also makes it possible, subsequently, to elucidate associations that may exist between component intervention practices and specific features of the context in which the intervention is implemented (Chorpita \& Daleiden, 2009).

The Distillation and Matching Model has been used in a number of studies over the past decade. While all these studies have demonstrated the process of distillation, few have 
extended their analyses further to conduct the matching component. Nonetheless, the model has been successfully applied to distill the practice and program elements across interventions for conflict-affected children and youth (Brown et al., 2017), children with disruptive behavior problems (Garland et al., 2008), youth at risk for out-of-home care (Lee et al., 2014), and families with multiple problems (Visscher et al., 2018) the latter of which also set out to develop a taxonomy similar in process to that which will be presented in this paper. The most comprehensive study to date, incorporating both the distillation and matching components of the model was that conducted by Chorpita and Daleiden who reviewed 322 randomized controlled trials spanning 615 tested interventions for childhood problems (Chorpita \& Daleiden, 2009). Their findings indicate that these interventions were comprised of a large number of varied practice elements which were organized primarily according to the contextual variable of diagnosis, within which some further delineations according to age and ethnicity could be identified. For example, the profile of practice elements for the treatment of depressed mood is distinguishable from that of attention deficit/hyperactivity, but even within the depressed mood category, distinctions in practice element profiles can be observed between Black children with depressed mood compared to Hispanic children with depressed mood (Chorpita \& Daleiden, 2009). The implication of this finding is that the specific clinical approach to intervening in depressed mood may need to be different depending on the ethnic background of the client presenting for treatment. This example highlights the potential of the distillation and matching model to not only codify clinical strategies, but to tailor intervention packages to the specific conditions and characteristics of the clinical situation and client being treated.

As such, in the absence of existing analyses at the level of practice elements for attachment-based interventions, the present study set out to distill these clinical strategies for this body of effective interventions and to determine whether these strategies are organized 
differently depending on various contextual factors, such as age, ethnicity and more. Initially, the PracticeWise Psychosocial and Combined Treatments Coding Manual (PracticeWise, 2005) was adopted to code the identified studies and intervention manuals for the purposes of extracting data and generating a dataset. However, it became clear during the process of doing so that the existing codes for practice elements within the PracticeWise system were largely not applicable to the population of attachment-based interventions under investigation. While the use of the PracticeWise system was retained in full and in its original form, the need was identified to develop a coding supplement to use alongside this which could extract the practice elements within attachment-based interventions more specifically. The process of the development of this coding supplement - similar to that followed by Garland, Hawley, Brookman-Frazee and Hurlburt (Garland et al., 2008) as well as Visscher et al. (Visscher et al., 2018)—will be the focus of this paper. The results of the distillation and matching process will be presented separately, elsewhere. This paper will therefore describe the development of the taxonomy and lexicon of treatment operations for use as an instrument tailored specifically to code practice elements within attachment-based interventions in order to make it possible to comment on what features are characteristic of, and common across, such interventions. This taxonomic tool will facilitate answering, in the context of attachment-based interventions, the question posed by Chorpita et al. (2005, p. 9): "...how [does] the clinician, faced with a particular client in a particular context...choose the most appropriate...approach?". The taxonomy will be graphically represented here, and a textual demonstration of the use and application of the taxonomy is presented by way of illustration. The taxonomy itself will also be included as an appendix to facilitate its accessibility to researchers and clinicians.

\section{Methods}

\section{Identification of attachment-based interventions}


Similar to the strategy adopted by Garland and colleagues (Garland et al., 2008), the primary search strategy used to identify publications was to hand-search the reference lists of existing published meta-analyses and systematic reviews of attachment-based interventions (Bakermans-Kranenburg et al., 2003; Bakermans-Kranenburg et al., 2005; Barlow et al., 2016; Facompré et al., 2018; Letourneau et al., 2015; Mortensen \& Mastergeorge, 2014; Mountain et al., 2017; Wright \& Edginton, 2016; Wright et al., 2017). This was supplemented with searches within scholarly databases such as MEDLINE, PsychArticles, PsychInfo, PubMed and Google Scholar as well as consultations with experts to ensure that the relevant publications had been identified. 230 publications were initially identified through these search strategies (see Fig 1). After duplicate publications were removed, the full texts of 135 articles were screened for eligibility using the inclusion criteria outlined in Table 1.

\section{Table 1}

Criteria for the inclusion of studies.

\begin{tabular}{|c|c|}
\hline Publication Period & January 1998 - December 2018 \\
\hline $\begin{array}{l}\text { Population, or } \\
\text { participants and } \\
\text { conditions of interest }\end{array}$ & Studies including children of any age. \\
\hline $\begin{array}{l}\text { Interventions or } \\
\text { exposures }\end{array}$ & $\begin{array}{l}\text { Caregiver-focused interventions aimed at addressing attachment } \\
\text { security/organisation in the child, and/or caregiver sensitivity. }\end{array}$ \\
\hline Outcomes of interest & $\begin{array}{l}\text { 1. Attachment security or organisation using any method of } \\
\text { assessment. } \\
\text { 2. Caregiver sensitivity using any method of assessment. }\end{array}$ \\
\hline Setting & Any \\
\hline Study designs & $\begin{array}{l}\text { Randomised controlled/clinical trials (RCT)_only studies which } \\
\text { included a process of full randomisation of participants into an } \\
\text { intervention group and at least one control condition. (Partially } \\
\text { randomised studies were excluded i.e. a study that randomly assigned } \\
\text { participants to an intervention group and a control group, but also } \\
\text { included a non-randomised comparison group in the analysis.) }\end{array}$ \\
\hline Language & Only articles published in English. \\
\hline
\end{tabular}


Following the eligibility screening, a total of 66 publications remained for inclusion in the coding process. These 66 publications covered a total of 49 distinct attachment-based intervention protocols. In addition, intervention developers were contacted in order to request the version of the manuals used for the interventions reported on in the published studies -17 $(35 \%)$ such full intervention manuals were obtained and included in the study, with developer consent. According to Chorpita and Daleiden (2009), coding of practice elements should occur using the best available description of the intervention; this is usually the intervention manual but in the absence thereof the description of the intervention as provided in the published paper is an acceptable alternative. This approach was adopted in the current process of developing the supplemental coding system — where the manual was not available for coding, the description of the intervention provided in published articles was coded. 


\section{Figure 1}

Systematic search strategy to identify studies.
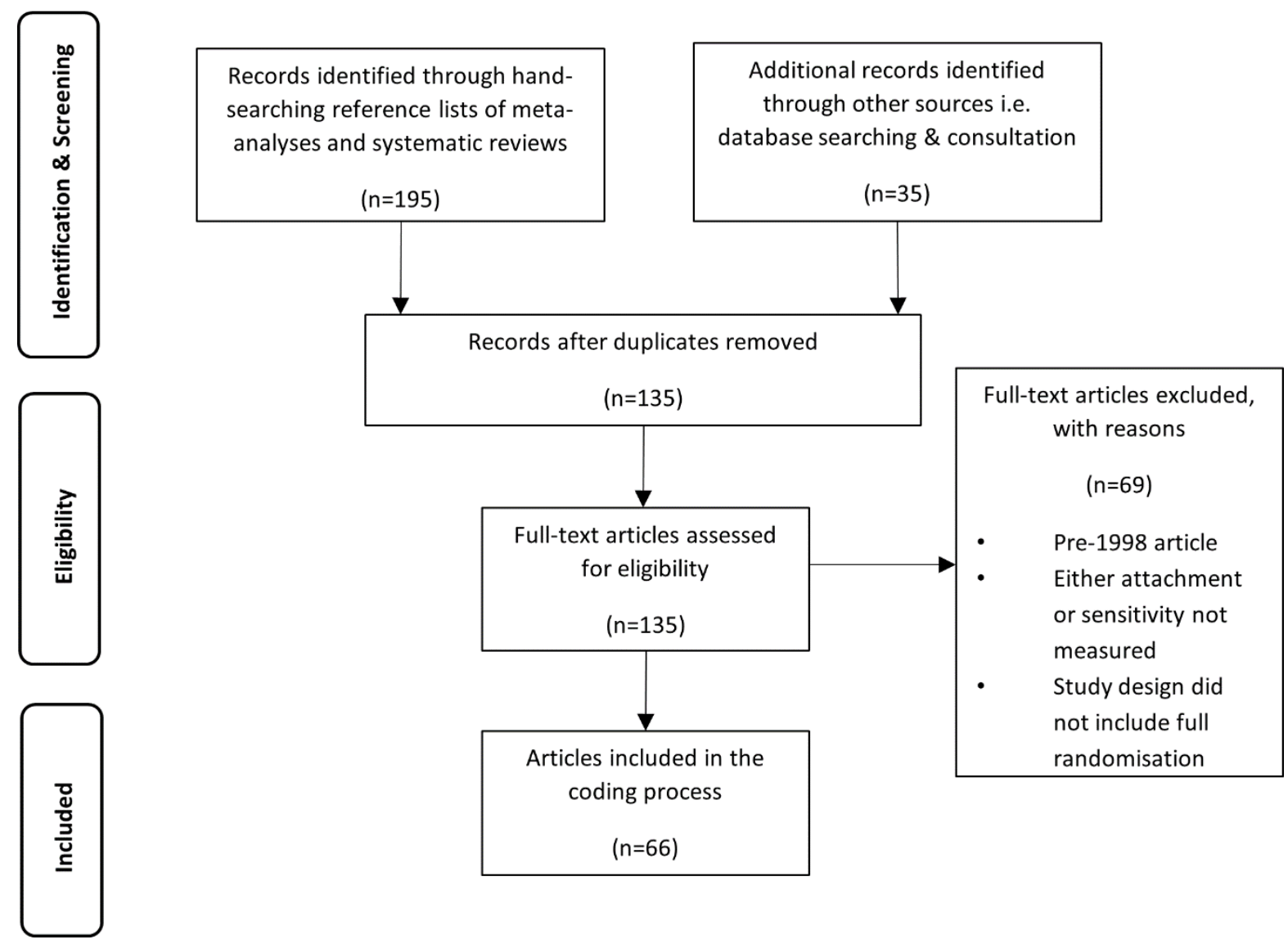

Articles included in the coding process

$(n=66)$

\section{Procedures}

In order to generate practice element codes, two primary coders (AM and PS) independently conducted an in-depth and detailed reading of the intervention descriptions/manuals to familiarize themselves with the content of the interventions. A procedure similar to that described by Braun and Clarke (2006) was then initiated by engaging an inductive open coding process in order to identify patterns of recurring techniques across all interventions. Here, during a line-by-line reading of each intervention description, significant instances were assigned a code that captured the essence of the technique. The identification of these techniques and the open coding thereof was guided firmly by the definition of a practice 
element as "a discrete clinical technique or strategy (e.g., "time out," "relaxation") used as part of a larger intervention plan (e.g., a manualized treatment program for youth depression)" (Chorpita et al., 2005, p. 11). Notes were taken during this process regarding the meanings of codes in order to facilitate the later development of definitions of practice elements. This inductive coding process was conducted independently by each coder, at first, and then discussed to reach consensus where there was significant deviation. After coding 11 studies in this manner, no new codes emerged and coders decided on data saturation. Following data saturation, an initial grouping into thematic areas was attempted, to provide insight into intervention foci and techniques.

At this stage, preliminary operational definitions for the practice element codes subsumed under each theme were developed by the first author based on notes taken during the open coding process. The operational definitions were drafted to capture, in a comprehensive manner, the vicissitudes within each practice element so as to facilitate its reliable use by others. A spreadsheet was created that contained each overall thematic area, the practice element codes subsumed under each, and their respective operational definitions. This spreadsheet served as the first draft of the Supplemental Practice Element Coding System for Attachment-based Interventions.

For the purposes of validation, the draft coding system was independently reviewed by CS, who had not been involved in the inductive coding process. This review led to further iterative minor adjustments, wherein practice elements codes were either split or merged, and the operational definitions were adjusted to provide further clarity and specificity by reducing ambiguity and thus enhancing its utility for future users of the coding system. A revised version of the taxonomy was organized according to the five themes listed in Table 2 and contained a total of 16 practice element codes and their respective definitions. The preliminary average interrater reliability for the Supplemental Practice Element Coding 
System for Attachment-based Interventions, calculated on the pre-consensus coding of the full set of intervention descriptions and protocols, fell within the good/substantial range $(\kappa=$ .7) according to criteria proposed by Landis and Koch (1977) for Cohen's Kappa.

\section{Table 2}

Practice Element Thematic Areas.

\begin{tabular}{ll}
\hline Thematic Area & General Description \\
\cline { 1 - 1 } $\begin{array}{l}\text { Modelling } \\
\text { Parentice elements involving the direct demonstration of desired caregiving } \\
\text { behaviors by either a therapist/trained intervenor, or peer (e.g. a fellow } \\
\text { caregiver in the context of a group intervention). } \\
\text { (Behavior-focused) }\end{array}$ & $\begin{array}{l}\text { Practice elements focusing on the facilitation of parent-child interactive } \\
\text { behavior. }\end{array}$ \\
$\begin{array}{l}\text { Mental } \\
\text { Representations } \\
\text { (Representation- } \\
\text { focused) }\end{array}$ & $\begin{array}{l}\text { Practice elements focusing on the development/revision of the caregiver's } \\
\text { mental representations. }\end{array}$ \\
$\begin{array}{l}\text { Education \& } \\
\text { Guidance }\end{array}$ & $\begin{array}{l}\text { Practice elements in which there is a distinct focus on (psycho)education } \\
\text { related either to child development, generally, or more specifically to } \\
\text { interaction. }\end{array}$ \\
Other & $\begin{array}{l}\text { Additional practice elements that recurred regularly but could not be } \\
\text { categorized, thematically (e.g. skin-to-skin) }\end{array}$ \\
\hline
\end{tabular}

\section{Results}

Fig 2 outlines the five themes subdivided into their respective practice element codes (in circles). The full taxonomy, including practice element definitions, has been included as Supplementary Material to this paper. 
Figure 2.

Practice element codes subdivided by theme.

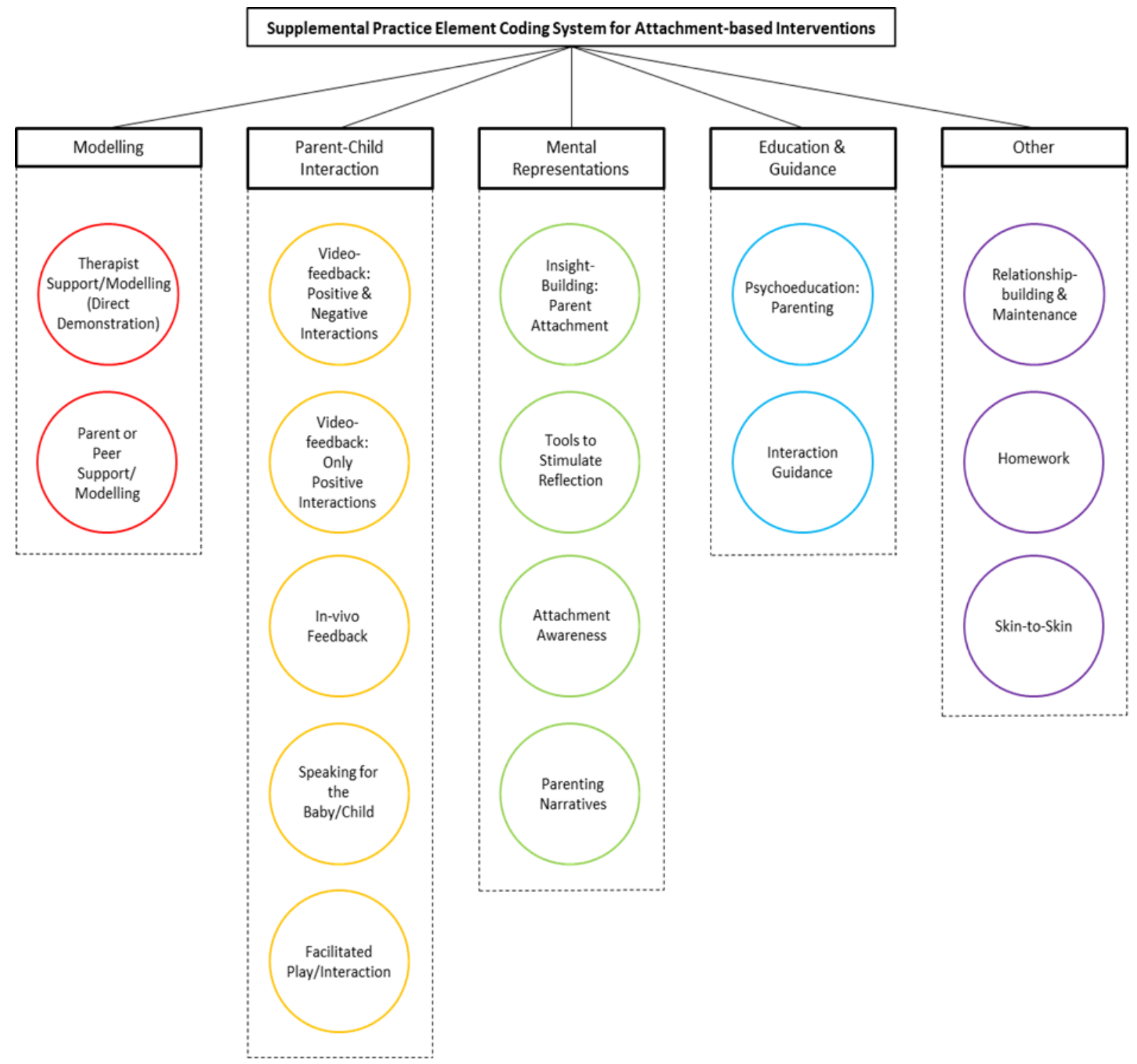

Although the Supplemental Practice Element Coding System for Attachment-based Interventions can be used independently, it was designed as both an extension and refinement of the existing PracticeWise system (PracticeWise, 2005) in order to enhance its utility in relation to this class of interventions. The PracticeWise system is organized into sections which allows for the extraction of data from studies at the overall study level, group level (treatment versus control) and protocol level (intervention content). Information extracted at 
the study and group level are used to build a dataset of the contextual elements of the study being coded including variables such as age, gender, ethnicity, diagnosis, as well as intervention-related characteristics such as therapist, setting, format, session length, session frequency and duration of intervention. Also coded here are the results of the study as they pertain to the target variables. The third section within the PracticeWise system relates to the extraction of data at the level of the intervention protocol (i.e practice elements). The system lists 73 predetermined practice elements that are coded if they feature in the respective intervention protocol. Provision is made for the addition of up to three additional practice elements should this be required in any given instance. The Protocol Coding Sheets are then used to build a second dataset of the practice elements evident across all coded interventions. The Supplemental Practice Element Coding System for Attachment-based Interventions extends and refines the Protocol Coding Sheet of the PracticeWise system. The practice elements proposed in the Supplement presented in this paper extend the PracticeWise system in that there are some elements which have been added and thus which lie at the same hierarchical level as those in the existing system. However, there are other elements which can be regarded as a refinement of existing practice elements in order to account for its unique content within attachment-based interventions. As an example, PsychodeducationParenting may be viewed as a refinement of the Psychoeducation-Caregiver practice element in the PracticeWise system. However, Speaking for the Baby/Child is an addition and, therefore, an extension in that such a practice element is currently not included in the PracticeWise system and cannot be subsumed within any of the existing practice elements using the current definitions.

To demonstrate how the coding supplement can be used to code the practice elements of an attachment-based intervention, the Attachment and Biobehavioral Catch-up (ABC) intervention was chosen as a worked example. For purposes of demonstration, the example in 
Table 3 is based on the intervention description published by Bick and Dozier (2013) rather

than the unpublished intervention manual. A profile is built for an intervention by coding,

dichotomously, the presence or absence of each practice element regardless of whether it

appears only once in an intervention or at multiple points. In the current version of the

taxonomy this is done by selecting the appropriate tick box on the PDF form of the coding

sheet which allows electronic scoring and data extraction in a way that is similar to the

standard PracticeWise coding sheets (see Appendix).

\section{Table 3}

Demonstration of application of the coding supplement.

Intervention Description"
"The last portion of the session is dedicated to
"video-feedback" on mother-infant interactions
over the previous session. During the feedback
portion of the session, parent trainers highlight
instances in which foster mothers either
successfully responded to their foster infant's
need or may have had difficulty doing so due to
the foster infant's alienating behaviour."

"Similar to previous sessions, parent trainers provide "in the moment" feedback during the actual parent-child interactions..."

"During these sessions, parent trainers help foster mothers think through their experiences with their own caregivers, and reflect on how these experiences may shape their reactions to their foster infant's bids for nurturance and sensitive care."

\section{Application}

Video-feedback: Positive and Negative

Interactions - In this extract the description states " "video-feedback' on mother-infant interactions". As such, one of two "videofeedback" codes would apply. Although the description refers to instances in which the mother successfully responds to the infant, which would be coded as "positive", it also refers to instances in which she may have had difficulty doing so, which would be coded as negative. Hence, both positive and negative interactions are commented on using video feedback which leads to coding the presence of the practice element Video-feedback: Positive and Negative Interactions.

In-vivo Feedback-In this example, the description refers to feedback as it happens in the moment while the parent-child interaction is taking place during the intervention session. This aligns with the definition of the In-vivo Feedback practice element, which refers to feedback to the parent by a therapist or intervenor based on the here-and-now interaction between parent and child as it happens during an intervention session.

Insight-building: Parent Attachment-The definition of this practice element refers to techniques used in an intervention in which the parent is assisted in better understanding how their own experiences of being parented impact on how they behave as parents towards their 
"During Session 9, parent trainers discuss the benefits of engaging in close physical contact with their foster infants. After reviewing research related to the importance of touch and cuddling for promoting infants' biobehavioral regulation, well-documented among groups of infants born in high-risk environments..." own children. Given that this technique addresses how parents have engaged with their own attachment histories, it deals in essence with parental attachment representations. Furthermore, as the description here states, this aspect deals directly with helping parents think about those representations and experiences and how these may be influencing their own parenting practice, which is why the practice element Insight-building: Parent Attachment is coded in this instance.

Psychoeducation: Parenting-This practice element is exclusively educational and does not directly involve the enhancement of insight into personal circumstances. The intention here is to enhance knowledge of a number of domains of parenting, in general. The knowledge is not necessarily a commentary on the parent-child relationship in the moment, but does have relevance to this relationship, more generally. In this description, for example, reference is made to discussing the benefits of maintaining physical contact with infants and reviewing research that relates to this and its impact on regulation. The discussion here does not relate directly to the index parent's parenting practices as observed by the intervenor. This particular element refers to an exchange of information or provision of guidance relevant to parenting practices, in general, therefore indicating the presence of the practice element Psychoeducation: Parenting.

Note: ${ }^{1}$ Extracted from Bick and Dozier (Bick \& Dozier, 2013)

Therefore, based exclusively on the limited extracts presented above, the coding sheet would be completed as presented in Fig 3, reflecting the four practice elements that have been coded for the $\mathrm{ABC}$ according to the Supplemental Practice Element Coding System for Attachment-based Interventions. 


\section{Figure 3}

Demonstration of completed coding sheet.

\begin{tabular}{|c|c|c|}
\hline Theme & Practice Element Code & $\begin{array}{c}\text { Select only } \\
\text { those that } \\
\text { apply }\end{array}$ \\
\hline \multirow[b]{2}{*}{ Modelling } & Therapist Support/Modelling (Direct Demonstration) & $\square$ \\
\hline & Parent or Peer Support/Modelling & $\square$ \\
\hline \multirow{5}{*}{$\begin{array}{l}\text { Parent-Child Interaction } \\
\text { (Behavior-focused) }\end{array}$} & Video-feedback using both Positive and Negative Interactions & 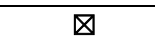 \\
\hline & Video-feedback using only Positive Interactions & $\square$ \\
\hline & In-vivo Feedback & 凶 \\
\hline & Speaking for the Baby/Child & $\square$ \\
\hline & Facilitated Play/Interaction & $\square$ \\
\hline \multirow{4}{*}{$\begin{array}{l}\text { Mental Representations } \\
\text { (Representation-focused) }\end{array}$} & Insight-building: Parent Attachment & 凶 \\
\hline & Tools to Stimulate Reflection & $\square$ \\
\hline & Attachment Awareness & $\square$ \\
\hline & Parenting Narratives & $\square$ \\
\hline \multirow{3}{*}{ Education \& Guidance } & Psychoeducation-Parenting & $\nabla$ \\
\hline & Interaction Guidance & $\square$ \\
\hline & Relationship-building and maintenance & $\square$ \\
\hline \multirow[t]{2}{*}{ Other } & Homework & $\square$ \\
\hline & Skin-to-skin Contact & $\square$ \\
\hline
\end{tabular}

\section{Discussion and Conclusion}

The novel coding system developed in this study is built on the procedures described in detail by Chorpita and colleagues (Chorpita \& Daleiden, 2009; Chorpita et al., 2005), using the PracticeWise system. Their proposal of a Distillation and Matching Model (DMM) to match profiles of practice elements with various characteristics of clinical interest (i.e. age, gender etc) in order to tailor nonpharmacological interventions for children's psychological challenges was the initial inspiration for attempting a similar approach with a specific focus on attachment-based interventions. Doing so makes it possible to implement flexible 
interventions that are structured around the specific needs and characteristics of a given client (Barth et al., 2014). Access to intervention resources and manuals is often limited, particularly in non-Western, developing, contexts where gaining such access can be costly and is not always feasible. The common elements approach holds promise through shifting the level of analysis and focus from whole intervention manuals, which may be inaccessible as intervention packages, to profiles of practice elements that span effective interventions. It may be more practical and efficient for individual clinicians to learn and master a range of core elements as opposed to several intervention protocols. Given that evidence-based treatments often share common clinical strategies that build on a shared set of clinical skills (Chorpita et al., 2007), learning those common strategies and prerequisite skills could serve to bypass the need to learn the full protocols while retaining the clinical utility thereof.

In order to apply this model successfully and meaningfully for the population of attachment-based interventions, the need was observed to supplement the existing practice element codes in the PracticeWise system with a subset of codes derived for attachmentbased interventions, as described above. A report on the initial application of the DMM using this supplement is currently in preparation for publication elsewhere. It is, however, acknowledged that the Supplement may still be regarded as a work-in-progress and that updates and revisions are therefore still possible (and perhaps even necessary given innovation in the field and the potential introduction of new clinical approaches). This Supplement allows for the extension and deepening of research on attachment-based interventions along avenues that may previously not have been possible at this level of specificity (i.e. individual practice elements). For instance, making it possible to identify component elements allows for dismantling studies to be conducted which can identify the so-called 'active ingredients' of interventions (Cuijpers et al., 2019). Distilling the active ingredients within and across attachment-based interventions may in turn serve to facilitate 
trial designs and tailored interventions incorporating elements that have been established as the most active and clinically beneficial. It may also be possible, through the application of the Supplemental Practice Element Coding System for Attachment-based Interventions, to reimagine existing interventions. For instance, determining the level of difference or similarity between interventions based on their constituent elements could allow for potential merging of similarly constituted interventions, in practice. This could make it more manageable for clinicians to select an intervention strategy that is appropriate for, and targeted to, their respective clients' characteristics. This may also facilitate accessibility for clinicians by reducing the burden of training; instead of being trained in multiple different individual protocols, which is not always practically possible, training could occur in various techniques which could, in turn, be implemented according to the best possible approach as determined by the model. This, in turn, could serve to streamline, simplify and standardize what is already available for implementation which has the potential to significantly impact clinical practice.

\section{Acknowledgements}

The authors wish to thank the developers of the interventions for sharing their respective intervention manuals for the purpose of the present study.

\section{Funding}

The financial assistance of the National Research Foundation (NRF) towards this research is hereby acknowledged. Opinions expressed and conclusions arrived at, are those of the authors and are not necessarily to be attributed to the NRF. This study was made possible by the Vrije Universiteit Amsterdam-NRF Desmond Tutu Doctoral Programme which is coordinated by the South Africa-Vrije Universiteit Strategic Alliance (SAVUSA). 


\section{Supporting Information}

Supplementary Material: S1 Appendix-Supplemental Practice Element Coding System

\section{References}

Ainsworth, M. D. S., Blehar, M. C., Waters, E., \& Wall, S. (1978). Patterns of attachment: A psychological study of the Strange Situation. Lawrence Erlbaum.

Bakermans-Kranenburg, M. J., van IJzendoorn, M. H., \& Juffer, F. (2003). Less is more: Meta-analyses of sensitivity and attachment interventions in early childhood. Psychological Bulletin, 129(2), 195-215. https://doi.org/10.1037/0033-2909.129.2.195

Bakermans-Kranenburg, M. J., van IJzendoorn, M. H., \& Juffer, F. (2005). Disorganized infant attachment and preventive interventions: A review and meta-analysis. Infant Mental Health Journal, 26(3), 191-216. https://doi.org/10.1002/imhj.20046

Barlow, J., Bennett, C., Midgley, N., Larkin, S. K., \& Wei, Y. (2016). Parent-infant psychotherapy: A systematic review of the evidence for improving parental and infant mental health. Journal of Reproductive and Infant Psychology, 34(5), 464-482. https://doi.org/10.1080/02646838.2016.1222357

Barth, R. P., Kolivoski, K. M., Lindsey, M. A., Lee, B. R., \& Collins, K. S. (2014). Translating the common elements approach: Social work's experiences in education, practice, and research. Journal of Clinical Child and Adolescent Psychology, 43(2), 301-311. https://doi.org/10.1080/15374416.2013.848771

Bick, J., \& Dozier, M. (2013). The effectiveness of an attachment-based intervention in promoting foster mothers' sensitivity toward foster infants. Infant Mental Health Journal, 34(2), 95-103. https://doi.org/10.1002/imhj.21373 
Bowlby, J. (1969/1997). Attachment and loss: Volume 1. Attachment. Pimlico.

Braun, V., \& Clarke, V. (2006). Using thematic analysis in psychology. Qualitative Research in Psychology, 3(2), 77-101. https://doi.org/10.1191/1478088706qp063oa

Brown, F. L., de Graaff, A. M., Annan, J., \& Betancourt, T. S. (2017). Annual research review: Breaking cycles of violence - a systematic review and common practice elements analysis of psychosocial interventions for children and youth affected by armed conflict. Journal of Child Psychology and Psychiatry, 58(4), 507-524. https://doi.org/10.1111/jcpp.12671

Cassidy, J., Brett, B. E., Gross, J. T., Stern, J. A., Martin, D. R., Mohr, J. J., \& Woodhouse, S. S. (2017). Circle of Security-Parenting: A randomized controlled trial in Head Start. Development and Psychopathology, 29(2), 651-673. https://doi.org/10.1017/S0954579417000244

Chorpita, B. F., Becker, K. D., \& Daleiden, E. L. (2007). Understanding the common elements of evidence-based practice: Misconceptions and clinical examples. Journal of the American Academy of Child and Adolescent Psychiatry, 46(5), 647-652. https://doi.org/10.1097/chi.0b013e318033ff71

Chorpita, B. F., \& Daleiden, E. L. (2009). Mapping evidence-based treatments for children and adolescents: Application of the distillation and matching model to 615 treatments from 322 randomized trials. Journal of Consulting and Clinical Psychology, 77(3), 566-579. https://doi.org/10.1037/a0014565

Chorpita, B. F., Daleiden, E. L., \& Weisz, J. R. (2005). Identifying and selecting the common elements of evidence based interventions: A Distillation and Matching Model. Mental Health Services Research, 7(1), 5-20. https://doi.org/10.1007/s11020-005-1962-6 
Cuijpers, P., Cristea, I. A., Karyotaki, E., Reijnders, M., \& Hollon, S. D. (2019). Component studies of psychological treatments of adult depression: A systematic review and meta-analysis. Psychotherapy Research, 29(1), 15-29. https://doi.org/10.1080/10503307.2017.1395922

Dozier, M., Lindheim, O., Lewis, E., Bick, J., Bernard, K., \& Peloso, E. (2009). Effects of a foster parent training program on young children's attachment behaviors: Preliminary evidence from a randomized clinical trial. Child and Adolescent Social Work Journal, 26(4), 321-332. https://doi.org/10.1007/s10560-009-0165-1

Facompré, C. R., Bernard, K., \& Waters, T. E. A. (2018). Effectiveness of interventions in preventing disorganized attachment: A meta-analysis. Development and Psychopathology, 30(1), 1-11. https://doi.org/10.1017/S0954579417000426

Garland, A. F., Hawley, K. M., Brookman-Frazee, L., \& Hurlburt, M. S. (2008). Identifying common elements of evidence-based psychosocial treatments for children's disruptive behavior problems. Journal of the American Academy of Child and Adolescent Psychiatry, 47(5), 505-514. https://doi.org/10.1097/CHI.0b013e31816765c2

Groh, A. M., Fearon, R. M. P., van IJzendoorn, M. H., Bakermans-Kranenburg, M. J., \& Roisman, G. I. (2017). Attachment in the early life course: Meta-analytic evidence for its role in socioemotional development. Child Development Perspectives, 11(1), 7076. https://doi.org/10.1111/cdep.12213

Juffer, F., Bakermans-Kranenburg, M. J., \& van IJzendoorn, M. H. (2017). Pairing attachment theory and social learning theory in video-feedback intervention to promote positive parenting. Current Opinion in Psychology, 15, 189-194. https://doi.org/10.1016/j.copsyc.2017.03.012 
Landis, J. R., \& Koch, G. G. (1977). The measurement of observer agreement for categorical data. Biometrics, 33(1), 159-174.

Lee, B. R., Ebesutani, C., Kolivoski, K. M., Becker, K. D., Lindsey, M. A., Brandt, N. E., Cammack, N., Strieder, F. H., Chorpita, B. F., \& Barth, R. P. (2014). Program and practice elements for placement prevention: A review of interventions and their effectiveness in promoting home-based care. American Journal of Orthopsychiatry, 84(3), 244-256. https://doi.org/10.1037/h0099811

Lemmens, L., Muller, V., Arntz, A., \& Huibers, M. J. H. (2016). Mechanisms of change in psychotherapy for depression: An empirical update and evaluation of research aimed at identifying psychological mediators. Clinical Psychology Review, 50, 95-107. https://doi.org/10.1016/j.cpr.2016.09.004

Letourneau, N., Tryphonopoulos, P., Giesbrecht, G., Dennis, C. L., Bhogal, S., \& Watson, B. (2015). Narrative and meta-analytic review of interventions aiming to improve maternal-child attachment security. Infant Mental Health Journal, 36(4), 366-387. https://doi.org/10.1002/imhj.21525

Mortensen, J. A., \& Mastergeorge, A. M. (2014). A meta-analytic review of relationshipbased interventions for low-income families with infants and toddlers: Facilitating supportive parent-child interactions. Infant Mental Health Journal, 35(4), 336-353. https://doi.org/10.1002/imhj.21451

Mountain, G., Cahill, J., \& Thorpe, H. (2017). Sensitivity and attachment interventions in early childhood: A systematic review and meta-analysis. Infant Behavior and Development, 46, 14-32. https://doi.org/10.1016/j.infbeh.2016.10.006 
Oxford, M. L., Spieker, S. J., Lohr, M. J., \& Fleming, C. B. (2016). Promoting First Relationships: Randomized trial of a 10-week home visiting program with families referred to child protective services. Child Maltreatment, 21(4), 267-277. https://doi.org/10.1177/1077559516668274

PracticeWise. (2005). Psychosocial and combined treatments coding manual. Author.

Visscher, L., Evenboer, K. E., Jansen, D. E. M. C., Scholte, R. H. J., Knot-Dickscheit, J., Veerman, J. W., Reijneveld, S. A., \& van Yperen, T. A. (2018). Identifying practice and program elements of interventions for families with multiple problems: The development of a taxonomy. Children and Youth Services Review, 95, 64-70. https://doi.org/10.1016/j.childyouth.2018.10.030

Wright, B., \& Edginton, E. (2016). Evidence-based parenting interventions to promote secure attachment: Findings from a systematic review and meta-analysis. Global Pediatric Health, 3, 1-14. https://doi.org/10.1177/2333794x16661888

Wright, B., Hackney, L., Hughes, E., Barry, M., Glaser, D., Prior, V., Allgar, V., Marshall, D., Barrow, J., Kirby, N., Garside, M., Kaushal, P., Perry, A., \& McMillan, D. (2017). Decreasing rates of disorganised attachment in infants and young children, who are at risk of developing, or who already have disorganised attachment. A systematic review and meta-analysis of early parenting interventions. PLoS One, 12(7), Article e0180858. https://doi.org/10.1371/journal.pone.0180858 